\title{
Cerebral epidural empyema due to Bartonella henselae: a case report
}

\author{
Stéphanie Matta ${ }^{1}$, Audrey Rousseau ${ }^{2}$, Rachel Chenouard ${ }^{1}$, Carole Lemarié ${ }^{1}$, Matthieu Eveillard ${ }^{1,4}$, \\ Marie Kempf ${ }^{1,4}$, Rafaël Mahieu ${ }^{3,4}$ and Hélène Pailhoriès ${ }^{1,5^{*}}$
}

\begin{abstract}
Background: Cat scratch disease frequently involves a benign, self-limited disease. Neurological forms associated with Bartonella henselae are uncommon, consisting mostly in neuroretinitis, encephalitis and meningitis. Cerebral epidural empyema has never described.
\end{abstract}

Case presentation: An adult patient was hospitalized for isolated headaches. Magnetic Resonance Imaging (MRI) identified typical features of cerebral epidural empyema. The diagnosis of $B$. henselae was performed incidentally by 165 rDNA gene sequencing on the abscess fluid, and confirmed by specific qPCR. We report here the first case, to our knowledge, of cerebral epidural empyema associated with B. henselae. Further follow-up visits allowed identifying frequent cat scratches on the scalp as the presumptive source of infection.

Conclusions: This case report alerts about such atypical clinical presentation, which requires an extensive clinical investigation. It also emphasizes on the usefulness of additional molecular diagnosis techniques in such CNS infection cases.

Keywords: Bartonella henselae, Cerebral epidural empyema, 16S rDNA gene sequencing, Cat Scratch Disease, Case report

\section{Background}

Bartonella henselae is a bacterium responsible for cat scratch disease (CSD), a zoonotic infectious disease usually transmitted to human by bites or scratches of domestic cat, its natural reservoir. Atypical clinical course in CSD occurs in a minority of cases (5-14\% of CSD) [1]. Among these atypical CSD, neurological manifestations associated with $B$. henselae are scarce, consisting mostly in neuroretinitis, meningoencephalitis and myelitis [2]. To our knowledge, cerebral epidural empyema has never been described in the literature. We report here the case of a cerebral epidural empyema due to $B$. henselae

\footnotetext{
*Correspondence: Helene.Pailhories@chu-angers.fr

${ }^{1}$ Laboratoire de Bactériologie, Institut de Biologie en Santé - PBH, CHU

Angers, 4 rue Larrey, 49933 Angers cedex, France

Full list of author information is available at the end of the article
}

diagnosed incidentally by $16 \mathrm{~S}$ rDNA gene sequencing on the abscess fluid.

\section{Case presentation}

An adult patient visited his general practitioner for a five-day history of high fever, diffuse abdominal pain and myalgia. Symptoms first disappeared and relapsed a few days later with the onset of gradual worsening headaches. A corticosteroid therapy was initiated considering sinusitis as a possible cause of the headaches, resulting in a complete resolution of all symptoms. After one week of treatment interruption, the headaches resumed and prompted the patient's admission to the hospital. He had no past medical history except for severe acne, tonsillectomy and surgery for a sinus polyp. No medication was reported at the time of admission. The patient worked as a welder and had a 5-year-old son. No exposure to animals was reported except for his cat. 
On hospital admission, clinical examination revealed a body temperature of $36.4{ }^{\circ} \mathrm{C}$. The patient reported isolated headaches. A cerebral computed tomography scan (CT) identified an epidural collection with contrast enhancement in the right frontoparietal area. The cerebral Magnetic Resonance Imaging (MRI) showed findings typical of a cerebral epidural empyema with FLAIR hyperintensities with gadolinium enhancing lesions (Fig. 1A, B). The thoraco-abdomino-pelvic CT was normal. The cerebrospinal fluid (CSF) analysis detected 30 WBCs $/ \mathrm{mm} 3$ including $85 \%$ of lymphocytes, with normal protein and glucose levels. The bacteriological cultures of the CSF were sterile after a 48-h incubation on sheep blood and chocolate agar plates in air atmosphere with $5 \% \mathrm{CO}_{2}$ as well as on Brain-Heart enrichment broth.

A surgical drainage of the epidural collection was performed, with bacteriological and pathological analyses. The direct examination with Gram staining was negative.
The bacteriological cultures remained sterile after 7 days of incubation on sheep blood agar in aerobic and anaerobic conditions, on chocolate agar in air with $5 \% \mathrm{CO}_{2}$ and in Schaedler and Brain-Heart enrichment broths.

Post-operative examination was normal with complete resolution of the headaches. The serum biochemical parameters were within reference range, the serum protein electrophoresis showed a restricted heterogeneity of gamma globulins, corresponding to a slightly irregular distribution of gamma globulins. No coagulation disorder was recorded and a normal level of thyroid stimulating hormone (TSH) was observed. The $\mathrm{C}$-reactive protein level was normal $(4 \mathrm{mg} / \mathrm{L}$, reference value $\leq 6 \mathrm{mg} / \mathrm{L})$. The serologies for human immunodeficiency virus, hepatitis $\mathrm{B}$ virus, hepatitis $\mathrm{C}$ virus, toxocariasis and hydatidosis were negative. Microscopic examination of the epidural collection by the pathologist (AR) showed a granulomatous inflammation associated with necrosis and granular

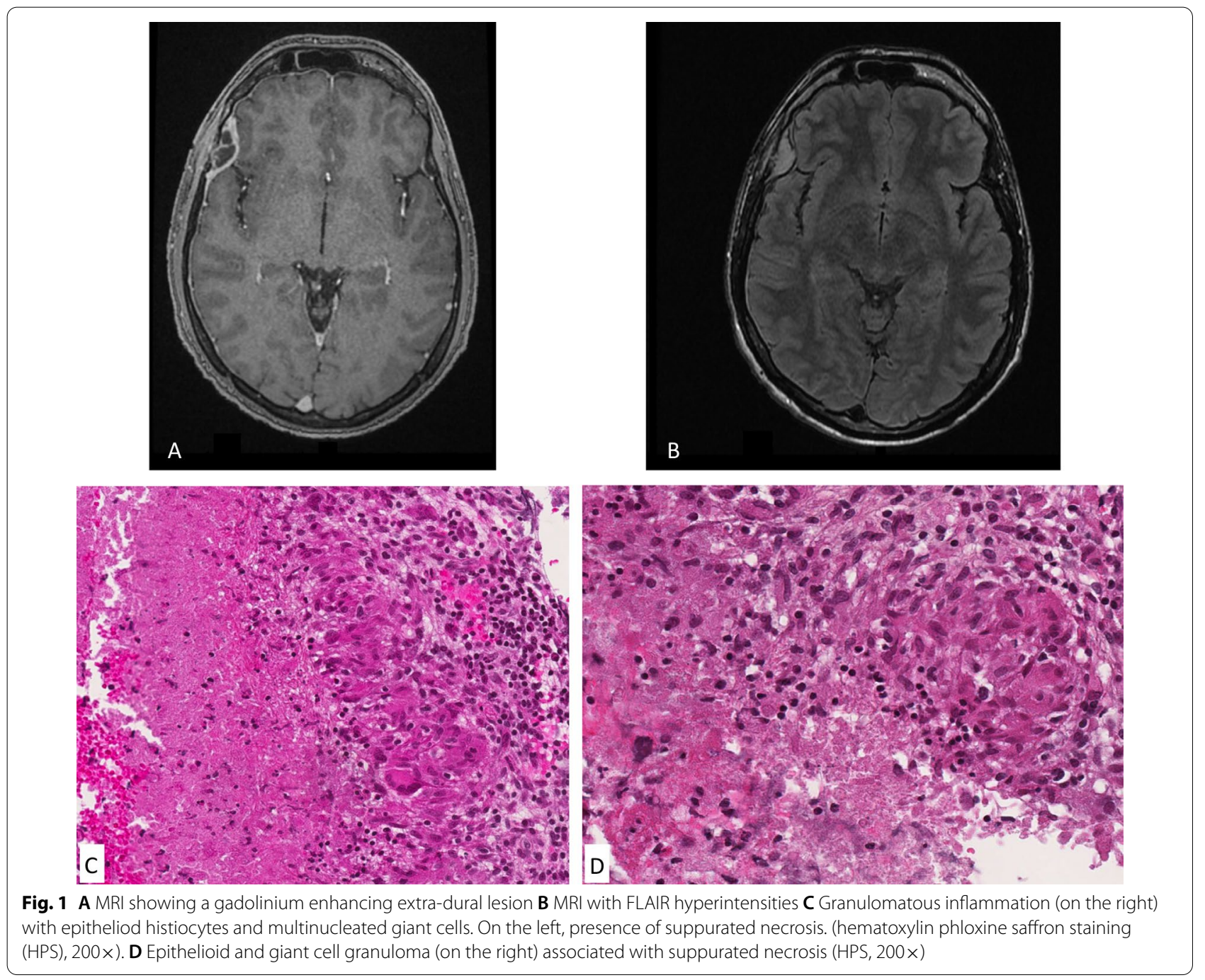


debris; neutrophils were recognizable. Grocott and Ziehl-Neelsen stainings were negative. There were no signs of malignancy (Fig. 1C, D). Meanwhile, 16S rDNA gene sequencing was performed on the abscess fluid with Sanger sequencing protocol, using the universal primer pair fD1 (5'-AGAGTTTGATCCTGGCTCAG-3') and rP2 (5'-ACGGCTACCTTGTTACGACTT-3') [3]. The result was analyzed using Basic Local Alignment Tool (BLAST) program through the National Center for Biotechnology Information (NCBI) server (https:// blast.ncbi.nlm.nih.gov/Blast.cgi). An identification of $B$. henselae was obtained. This result was confirmed by an in-house PCR targeting the rRNA 23S B. henselae gene (BH5 5'-GAGGCCCCTACCTCTGAAAGA-3' and BH6 5'-TCAAAGCCCACGGTGGA-3'). During the follow-up of the patient, it turned out that his cat made frequent scratches on his scalp.

A 1 month course of doxycycline (100 mg bid) was prescribed with no relapse during a two-year follow-up.

\section{Discussion and conclusions}

To the best of our knowledge, we report herein the first case of cerebral epidural empyema due to $B$. henselae described in the literature. This clinical description raises the question about the pathophysiology of Cat Scratch Disease (CSD) in this patient. Following inoculation of $B$. henselae, most patients with CSD present a benign self-limited infection. CSD is one of the leading causes of lymphadenopathy in children and adolescents but the exact interaction between the host immune system and the bacterium remains unknown. By invading endothelial cells with an increased production of vascular endothelial growth factor, $B$. henselae has also been associated with systemic diseases like endocarditis, hepatosplenic abscesses, retinopathy, uveitis, peliosis hepatitis, osteomyelitis, splenomegaly, pneumonia and glomerulonephritis [2]. Some of these manifestations have been mostly described in patients presenting an immunocompromised status like bacillary angiomatosis (BA) but the pathogenesis of each form of CSD remains poorly understood while documented BA in immunocompetent adults has also been reported $[4,5]$. Most neurological manifestations consist of neuroretinitis, encephalitis and meningitis. Peripheral neuropathy and acute hemiplegia have also been described during CSD. Diagnosis of CSDassociated encephalopathy may be challenging as normal MRI have been described despite the existence of neurological deficits [6]. Other manifestations include status epilepticus [7], cerebral vasculitis, with secondary cerebral infarction and necrosis [8], or brainstem encephalopathy with basal ganglia impairment [9]. Spinal epidural abscesses due to $B$. henselae have also been detailed in the literature [10-12]. In these cases, the spinal abscesses were all described in pediatric context (children from 5 to 10 years old) and associated with vertebral osteomyelitis. However, cerebral epidural empyema associated with $B$. henselae has never been reported in the literature. We suggest that this uncommon manifestation may reflect the atypical portal of entry of $B$. henselae in this patient with the cerebral epidural empyema corresponding to a localized manifestation of CSD. During a followup interview, the patient acknowledged that his cat had scratched his head on several occasions. Moreover, cerebral abscesses or empyema may be associated with local infection (sinusitis, acute medium otitis) or may appear in the post-operative setting. Haematogenous spread can be associated with brain abscesses and may complicate the clinical course of CSD endocarditis [13]. However, this source of contamination is unlikely in this case, the most likely transmission route being the scratches on the scalp. The emissary veins of the scalp could thus have played a role in the dissemination of the pathogen, providing a connection between the extracranial tissues and the intracranial transverse sinus, and possibly the epidural space. The initial prescription of corticosteroids without antibiotics could also contribute to the intracranial extension of the infection. The development of the granulomatous lesion in the epidural space was thus associated with the multiplication of $B$. henselae.

Because B. henselae is a fastidious bacterium that requires specific laboratory conditions, conventional growth techniques performed on cerebral samples may lack sensitivity for this pathogen. Overall, 7 to $53 \%$ of cultures of cerebral suppuration remain negative with conventional techniques. Because streptococci and anaerobes are the most commonly isolated microorganisms from empyema material and because most negative cultures are related to previous antibiotic treatment [14], the use of complex additional techniques may have been considered as futile. Recently, the causative bacteria involved in central nervous system (CNS) infections have been studied through a systematic comparison between 16S-rDNA-based next-generation sequencing and conventional techniques [15]. Molecular assays were able to identify a larger number of bacterial taxa compared to culture with the identification of several species infrequently recorded in these infections. Therefore, the absence of identification of infective microorganisms in a $\mathrm{CNS}$ infection requires an extensive investigation and the use of additional molecular-based techniques.

Most patients with CSD lymphadenitis experience a spontaneous resolution of their symptoms without specific antibiotic therapy. The fact that the antibiotic treatment may shorten the duration of symptoms or decrease the risk of systemic disease is debated in the literature [16, 17]. However, in a large cohort of 268 
patients with CSD lymphadenitis, antibiotic treatment was associated with a median duration of symptoms of 2.8 weeks compared with 14.5 weeks in patients without antibiotherapy [17]. Limited data are available for more severe forms of the disease but a combination of active agents against $B$. henselae is commonly used with a favorable outcome after a 4-week regimen [18]. A combination of doxycycline and rifampicin $[2,7,9,19]$ or azithromycin and rifampicin has been proposed for the management of CNS infections associated with $B$. henselae, in particular for encephalitis [7]. Because of their specific pathophysiology, intracranial epidural abscesses may be easier to treat than cerebral abscesses or encephalitis with the specific impact of surgical drainage. In our case, the evolution was favorable with surgical drainage and a one-month course of doxycycline.

This case report describes a first case of $B$. henselae cerebral epidural empyema. This emphasizes the potential unusual clinical presentation of $B$. henselae infection, which has been treated here by surgical drainage and appropriate antibiotic treatment. Furthermore, in CNS infections with negative microbiological culture results, the use of additional molecular techniques seems essential to the microbiological diagnosis.

\begin{abstract}
Abbreviations
BA: Bacillary angiomatosis; BLAST: Basic Local Alignment Tool; CNS: Central nervous system; CSD: Cat scratch disease; CSF: Cerebrospinal fluid; MRI: Magnetic resonance imaging; NCBI: National Center for Biotechnology Information.
\end{abstract}

\section{Acknowledgements}

None.

\section{Author's contributions}

AR participated in the management of the samples for analysis and in writing the manuscript. CL, ME, MK and RC participated in the management of the samples for analysis and in the revision of the manuscript. HP supervised the manuscript. SM wrote the manuscript. RM participated in the medical care and in the revision of the manuscript. All authors read and approved the final manuscript.

\section{Funding}

None.

\section{Availability of data and materials}

All data generated or analysed during this study are included in this published article.

\section{Declarations}

\section{Ethics approval and consent to participate}

A written informed consent from the patient has been obtained for the participation to this case report.

\section{Consent for publication}

A written informed consent from the patient has been obtained for the publication of this case report.

\section{Competing interests}

The authors declare that they have no competing interests.

\section{Author details}

'Laboratoire de Bactériologie, Institut de Biologie en Santé - PBH, CHU Angers, 4 rue Larrey, 49933 Angers cedex, France. ${ }^{2}$ Département de Pathologie
Cellulaire et Tissulaire, CHU d'Angers, 4, rue Larrey, 49933 Angers, France. ${ }^{3}$ Service de Maladies Infectieuses et Tropicales, CHU Angers, CHU, 4 rue Larrey, 49933 Angers cedex, France. ${ }^{4}$ CRCINA, INSERM, Université de Nantes, Université d'Angers, Angers, France. ${ }^{5}$ Laboratoire HIFIH, UPRES EA3859, SFR 4208, Université d'Angers, Angers, France.

Received: 15 September 2020 Accepted: 28 July 2021

Published online: 06 August 2021

References

1. Canneti B, Cabo-López I, Puy-Núñez A, et al. Neurological presentations of Bartonella henselae infection. Neurol Sci . 2019;40:261-8.

2. Angelakis E, Raoult D. Pathogenicity and treatment of Bartonella infections. Int J Antimicrob Agents. 2014;44:16-25.

3. Weisburg WG, Barns SM, Pelletier DA, Lane DJ. 165 ribosomal DNA amplification for phylogenetic study. J Bacteriol. 1991;173(2):697-703.

4. Tappero JW, Koehler JE, Berger TG, et al. Bacillary angiomatosis and bacillary splenitis in immunocompetent adults. Ann Intern Med. 1993;1 18:363-5.

5. KempfVA, Volkmann B, Schaller M, et al. Evidence of a leading role for VEGF in Bartonella henselae-induced endothelial cell proliferations. Cell Microbiol. 2001;3:623-32.

6. Samarkos M, Antoniadou V, Vaiopoulos AG, et al. Encephalopathy in an adult with cat-scratch disease. BMJ Case Rep. 2018. https://doi.org/10.1136/ bcr-2017-223647.

7. Schuster AL, Honeycutt TCB, Hamrick HJ. Status epilepticus due to cat scratch disease: recognition, diagnosis, and thoughts on pathogenesis. Pediatr Emerg Care. 2016;32:789-91.

8. Balakrishnan N, Ericson M, Maggi R, et al. Vasculitis, cerebral infarction and persistent Bartonella henselae infection in a child. Parasit Vectors. 2016;9:254

9. Genizi J, Kasis I, Schif A, et al. Effect of high-dose methyl-prednisolone on brainstem encephalopathy and basal ganglia impairment complicating cat scratch disease. Brain Dev. 2007;29:377-9.

10. Abdel-Haq N, Abuhammour W, Al-Tatari H, Asmar B. Disseminated cat scratch disease with vertebral osteomyelitis and epidural abscess. South Med J. 2005;98(11):1142-5.

11. Tasher D, Armarnik E, Mizrahi A, Liat BS, Constantini S, Grisaru-Soen G. Cat scratch disease with cervical vertebral osteomyelitis and spinal epidural abscess. Pediatr Infect Dis J. 2009;28(9):848-50.

12. Dornbos D 3rd, Morin J, Watson JR, Pindrik J. Thoracic osteomyelitis and epidural abscess formation due to cat scratch disease: case report. J Neurosurg Pediatr. 2016;25(6):713-6.

13. Brouwer MC, Tunkel AR, van de Beek D. Brain abscess. N Engl J Med. 2014;371:1758.

14. Osborn MK, Steinberg JP. Subdural empyema and other suppurative complications of paranasal sinusitis. Lancet Infect Dis. 2007;7:62-7.

15. Stebner A, Ensser A, Geißdörfer W, et al. Molecular diagnosis of polymicrobial brain abscesses with 165-rDNA-based next-generation sequencing. Clin Microbiol Infect . 2020;27(1):76-82.

16. Prutsky G, Domecq JP, Mori L, et al. Treatment outcomes of human bartonellosis: a systematic review and meta-analysis. Int J Infect Dis . 2013;17:e811-819.

17. Margileth AM. Antibiotic therapy for cat-scratch disease: clinical study of therapeutic outcome in 268 patients and a review of the literature. Pediatr Infect Dis J. 1992:11:474-8.

18. Berteau F, Mahieu R, Le Turnier P, et al. Hepatosplenic bartonellosis in immunocompetent adults: a case series and literature review. Eur J Clin Microbiol Infect Dis . 2020. https://doi.org/10.1007/s10096-020-03906-8.

19. Rolain JM, Brouqui P, Koehler JE, et al. Recommendations for treatment of human infections caused by Bartonella species. Antimicrob Agents Chemother. 2004;48:1921-33.

\section{Publisher's Note}

Springer Nature remains neutral with regard to jurisdictional claims in published maps and institutional affiliations. 